\title{
Beiträge zur Kenntnis des Phytoplanktons des Brienzersees
}

\section{Doctoral Thesis}

Author(s):

Flück, Hans

Publication date:

1926

Permanent link:

https://doi.org/10.3929/ethz-a-000092070

Rights / license:

In Copyright - Non-Commercial Use Permitted 


\title{
Beiträge zur Kenntnis desPhytoplanktons des Brienzersees
}

\author{
4 \\ Von der \\ Eidgenössischen Technischen Hochschule \\ in Zürich \\ zur Erlangung der Würde \\ eines Doktors der Natur- \\ wissenschaften genehmigte \\ Promotionsarbeit \\ Vorgelegt von \\ Hans Fluick, dipl. Apotheker \\ aus Brienz, Kt. Bern \\ Referent: \\ Prof.Dr.C.Schröter \\ Korreferent: \\ Prof. Dr. P. Jaccard
}

454.

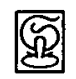

Graphische Werkstătten H. R. Sauerlănder \& Co. / Aarau 1926 
planktisch arm sind, muß in Beziehung stehen zu dem Detritusreichtum. Nur ist die Beziehung eine indirekte. Die erwähnten Seen mit viel mineralischem Detritus müssen Gletscherabwässer als Zuflüsse haben und diese zeigen meistens nicht einmal Spuren von Nitraten und Ammoniak. Daher zeigt das Milieuspektrum dieser Gewässer immer eine hochgradige N.Oligotrophie. Nun müssen aber auch die Katharoben Algen zum Aufbau ihrer Eiweißkörper Stickstoff haben und können daher nicht leben in diesen Gewässern ohne verwertbaren Stickstoff. Je kleiner also die Konzentration an diesem Stick$s$ t o f $\mathrm{f}$ ist, desto kleiner muß die Populationsdichte sein.

Als Reaktion auf die N.-, P- und Humusoligotrophie des Brienzersees müssen wir ebenfalls die Zusammensetzung des Planktons nach Arten auffassen. Auffällig ist da vor allem das vollständige Fehlen der Desmidiaceen, dann auch das geringe Vorkommen der Cyanophyceen und der Chlorophyceen. Von den Chlorophyceen ist nach Gams Botryococcus Braunii in seiner roten Form ein Zeichen von Eutrophie und in seiner grünen ein Zeichen von Oligotrophie. Diese Alge kommt nur in der grünen Form vor im Brienzersee.

Den Riesenanteil an Phytoplankton des Brienzersees machen die Diatomaceen und einen guten Teil auch noch die Flagellaten und Peridineen aus.

Die physikalischen Komponenten des Milieuspektrums sind für die Zusammensetzung des Phytoplanktons von wesentlich kleinerer Bedeutung als die chemischen.

\section{Ka p it el.}

\section{Zusammenfassung.}

1. Geographisch - geologische Verhältnisse. Nördlicher Alpenrandsee. Sein Becken, das nach Heim durch spätere Einsenkung des schon gebildeten Aaretales entstand, war nie mit dem Meere in Verbindung. Daher keine marinen Relikte möglich im Plankton. Form des Beckens wenig gegliedert. Uferentwicklung: Meist steiles Felsufer; Wysse schmal oder gar nicht entwickelt. Siedlungsverhältnisse: Umgebung und Einzugsgebiet sehr dünn bevölkert.

2. Klimatologie. Niederschlagshöhe kleiner als die des ganzen Einzugsgebietes. Mittlere Niederschlagsmengen: 1921 
$804 \mathrm{~mm}, 19221593 \mathrm{~mm}$. Mittlere Jahrestemperatur: $19219,1^{\circ}$, $19227,6^{\circ}$.

3. Hydrographische Verhältnisse. Große mittlere Tiefe $(176 \mathrm{~m})$, Oberfläche $29,2 \mathrm{~km}^{2}$, Volumen $5 \mathrm{~km}^{3}$. Große Wassermasse im Verhältnis zur Oberfläche; daher kleine Amplituden der physikalischen und chemischen Lebensbedingungen. $\mathrm{Zu}-$ flüsse zum größten Teile Gletscherflüsse mit viel Detritus. Abfluß durch die Aare künstlich reguliert.

4. Temperaturverhältnisse. Tropischer See nach Forel. Tiefste Grundtemperatur $4,8^{\circ}$, tiefste gemessene Temperatur $4,7^{\circ}$ bei $100 \mathrm{~m}$ Tiefe. Höchste gemessene Temperatur $24,9^{\circ}$. Noch nie zugefroren. Oberflächentemperaturen schwanken stark von Tag zu Tag. Sprungschicht im Sommer entwickelt. Homothermie tritt nicht ein. Temperaturperioden: 2 Perioden: 1. Sommerstagnation, 2. Winter-Teilzirkulation.

5. Transparenz. Vor allem durch Gletscherschlamm beeinflußt; im Sommer sehr klein (Minimum $45 \mathrm{~cm}$, Maximum im März 8,75 m).

6. Farbe. Winter: blau, schwach milchig getrübt; Sommer : grünblau, stark milchig getrübt. Planktogene Färbungen treten nie auf.

7. Chemismus. Sehr kleine Amplituden in vertikaler und zeitlicher Richtung. Sauerstoff: Größte Menge an der Oberfläche $8,17 \mathrm{ccm}$ pro Liter; kleinste Menge: $6,02 \mathrm{ccm}$ pro Liter. Gehalt nimmt im Winter von oben nach unten $a b$, im Sommer zu. Sauerstoffhaushalt ist thermisch bedingt. Freie Kohlensäure: Größte Menge an der Oberfläche 2,4 $\mathrm{mg}$ pro Liter; kleinste Menge: $0 \mathrm{mg}$ pro Liter. Gehalt nimmt immer von oben nach unten zu. Halbgebundene Kohlensäure: Größter Gehalt an der Oberfläche $32,6 \mathrm{mg}$ pro Liter; kleinster Gehalt: $30,1 \mathrm{mg}$ pro Liter. Härte: Größter Wert an der Oberfläche 5,0 deutsche Härtegrade; kleinster Wert 4,7 deutsche Härtegrade. Halbgebundene Kohlensäure und Härte nehmen immer von oben nach unten zu. Nitrate: Äußerst geringe Spuren.

8. Phytoplankton aus 5 Algenklassen.

3 Cyanophyceen:-Alle selten im See. Sommermaximum

8 Flagellaten: Häufig und wichtig. Maxima der einzelnen Arten verteilt durch das ganze Jahr.

5 Peridineen: 3 Arten davon häufig und wichtig. Sommermaxima

2i Diatomaceen: Wichtigste Klasse im Brienzersee. Maxima im Frühling und Herbst.

5 Chlorophyceen: Nur wenig entwickelt. Maxima verteilt. 
9. Zooplankton: Nicht eingehend untersucht. Auffällig ist Fehlen von Daphnia und Bosmina. Hauptrolle spielen Copepoden und Rotatorien.

10. Räumliche Verteilung des Planktons: Verteilung ist die Folge der Reaktionen des Planktons auf die Variationen der Lebensbedingungen.

a) Horizontale Verteilung: Lebensbedingungen im ganzen See in horizontaler Richtung gleichmäßig verteilt. Plankton ebenfalls gleichmäßig verteilt.

b) Vertikale Verteilung: Hauptlebenszone von 0 bis $10 \mathrm{~m}$. Absteigen bis $75 \mathrm{~m}$ (Flagellaten). Licht beeinflußt die vertikale Verteilung am meisten. Temperatur ebenfalls von Bedeutung, aber nicht so stark wie Licht. Chemismus ohne Einfluß; dagegen Einfluß des Planktons auf Chemismus (freie $\mathrm{CO}_{2}$, biogener Bicarbonatabbau).

10. Zeitliche Verteilung: Wird vor allem bedingt durch die Thermik. Licht weniger wichtig. Ohne Einfluß sind chemische Faktoren. Saisondimorphismen nicht in bedeutendem Maße vorhanden.

\section{Klassifikation des Sees:}

a) Nach Mili e uspektrum :

Oligotroph im allgemeinen.

$\mathrm{CO}_{2}$ und $\mathrm{Ca}$ mesotroph, 0 -meso- bis polytroph.

$\mathrm{N}, \mathrm{P}$ und Humus oligotroph.

b) Nach L e it s p e z i e s (pflanzengeographisch):

Typus Cyclotellasee nach Bachmann.

Unter-Typus cyanophyceenarmer Cyclotellasee mit den kleinen Cyclotellaarten, ohne Dinobryon sertularia.

Fehlen der Desmidiaceen, geringes Vorkommen der Cyanophyceen und Chlorophyceen sind Folge der N, P und HumusOligotrophie. 